\title{
PIV measurements of laminar flow around a hemisphere
}

\author{
A. Goharzadeh, L. Khezzar \& A. Molki \\ Department of Mechanical Engineering, The Petroleum Institute, \\ Abu Dhabi, UAE
}

\begin{abstract}
This paper presents an experimental study of laminar flow past a smoothed hemisphere using the Particle Image Velocimetry technique. The experimental setup consists of a rectangular channel, through which water flows over a $30-\mathrm{mm}$ diameter hemisphere mounted on a horizontal surface. From the measured velocity distribution around the hemisphere a physical insight into the flow is presented. Vertical and horizontal 2D velocity distributions are obtained for a constant Reynolds number of $\mathrm{Re}=800$, corresponding to a regime of laminar flow. Measurements revealed the three dimensional structure of the flow including a horseshoe vortex surrounding the hemisphere and arch-shaped vortices in the downstream region. Both instantaneous and average velocity distribution were studied. The location of the reattachment point, the separation line, and the reverse flow are identified and discussed.

Keywords: PIV, horseshoe vortex, hemisphere, dome.
\end{abstract}

\section{Introduction}

Hemispherical shapes are widely used in many industrial applications such as domed roofs in civil engineering to cover buildings or above-ground steel tanks to store fluids. Domes were used in hydraulic channels as a mechanism to generate and study the shedding of hairpin vortices in their wake [1]. Predicting the three dimensional structure of flow field around a hemisphere is challenging for engineers due to the complexity added by the curved surface. Therefore, in order to ensure proper design of such engineering structures, detailed knowledge of the flow structure around them is necessary. 
The flow around domes is characterized by large-scale three-dimensional motions with separation and multiple re-attachment points, which at times exhibit a highly unsteady behaviour. Thus, a small recirculating bubble exists around the front stagnation point and a horseshoe vortex starts to develop and envelopes parts of the dome. The wake is characterized by vortex shedding, flow separation, and reattachment. Several parameters affect the flow behaviour around domes: the dome shape, the Reynolds number (based on the approaching free stream velocity and dome diameter), the inflow conditions such as the approaching boundary layer shape, and the upstream-wall and dome surface conditions.

Most of the experimental studies were conducted under turbulent flow conditions. For example, Taniguchi et al. [2], Savory and Toy [3] and Cheng and $\mathrm{Fu}$ [4] conducted experimental investigations to study the effect of three different approaching boundary layers in addition to the effect of dome surface roughness on the mean pressure distribution and on the critical Reynolds number beyond which the pressure distribution becomes invariable. Tamai et al. [5] explored the formation and shedding of vortices from a dome in a water tunnel and determined the frequencies characterizing each phenomenon. As the maximum Reynolds number reached $10^{4}$, the Strouhal number had a value of 0.2 . Approaching the front side, successive expanding recirculating zones and the formation of the well-known horseshoe vortices were observed. The number of these structures increases with increasing Reynolds number until a critical value of about 3,000 , beyond which only one single recirculation zone persists with a constant size. Behind the dome, arched vortex tubes form and are shed individually or coalesce before shedding depending on a critical Reynolds number of 2,000. Using split-fiber probes and hot-wire anemometers Tavakol et al. [6] investigated the turbulent flow around a dome by obtaining axial velocity profiles and its root mean square (RMS) component. In their numerical investigations of laminar flow around a hemisphere, Kim and Choi [7] found that the separation angle is fixed at $90^{\circ}$ from the stagnation point at Reynolds numbers below 300 and that the flow loses its symmetry and steadiness with increasing Reynolds number with clear hairpin type vortex shedding. The onset of asymmetry and periodicity occur at a Reynolds number of between 170-180 and 190-200 respectively.

It appears that relatively few experimental studies were performed on laminar flow around hemispherical domes; in other words, the majority being confined to turbulent flows and where only point measurements were obtained. From these measurements, extrapolated but incomplete pictures of the flow structure were proposed. The flow around a dome is complex and three-dimensional and only planar velocity measurements can provide a more complete picture of the flow structure. Hence, the present work aims to fill this gap by reporting on velocity measurements and flow mapping obtained by using Particle Image Velocimetry (PIV) technique. The results are of sufficient accuracy to provide a benchmark for the testing of computational methods of such flows [7]. 


\section{Experimental facility and conditions}

\subsection{Experimental setup}

Experiments were conducted in a water tunnel in a closed circuit arrangement with the flow loop in a vertical configuration. The water tunnel consists of a reservoir, head tower, flow conditioning section, contraction section, square flume, and a downstream tower. The reservoir is of monolithic type, constructed from clear acrylic, and contains the working fluid. Flow is generated using a submersible pump (Grundfos Model KP 250). Flow conditioning is achieved by means of a perforated cylinder which distributes the flow to the head tower, a stainless steel perforated plate, and a series of acrylic screens. The contraction section has a 2.52:1 area ratio with a symmetrical cross section and analytically developed contours. The contraction guides the flow from the head tower to the square flume which acts as the experimental test section.

As shown in figure 1a, a smooth hemispherical dome with a diameter of 30 $\mathrm{mm}$ was mounted in the test section and placed $220 \mathrm{~mm}$ downstream of the contraction section. The dome was fabricated from acrylic using a conventional lathe machine and painted in black colour to minimize reflection. Flow velocity in the test section is estimated to vary from 15 to $300 \mathrm{~mm} / \mathrm{s}$ with an uncertainty of $+/-2 \%$ and is controlled using a ball valve.

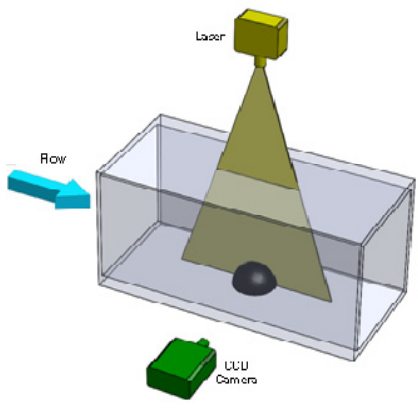

(a) Schematic view

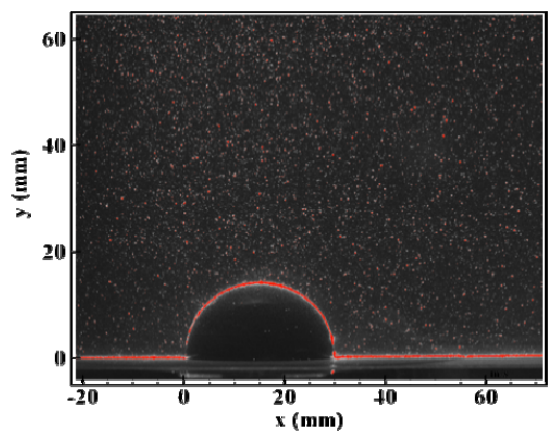

(b) Example of PIV image in $x-y$ plane with a large field of view $90 \times 70 \mathrm{~mm}^{2}$

Figure 1: Flow visualization system.

In the central region of the channel, the flow over the hemisphere is laminar and characterized by a constant fluid-based Reynolds number:

$$
R_{e}=\frac{U D}{v}
$$

where $\mathrm{U}=22 \mathrm{~mm} / \mathrm{s}$ is the bulk stream velocity in the horizontal direction, $\mathrm{D}=30$ $\mathrm{mm}$ the dome diameter and $v=0.8 \mathrm{~mm}^{2} / \mathrm{s}$ the kinematic viscosity of water at a constant temperature of $\mathrm{T}=30^{\circ} \mathrm{C}$ corresponding to the Reynolds number of $\mathrm{Re}=800$. 


\subsection{Flow conditions and measurement devices}

Using PIV, all flow field measurements were taken in two directions: (i) in the central vertical axial plane of the dome arrangement, fig. 1a, and (ii) in the horizontal plane near the bottom wall. To illuminate theses measurement planes, a laser sheet was projected vertically and horizontally, with a charge coupled device CCD camera positioned perpendicular to the plane to record particle motion, as illustrated in figure 1a. The water was seeded with polyamide particles tracers with an average diameter of $22 \mu \mathrm{m}$ and density of $1.016 \mathrm{~g} / \mathrm{cm}^{3}$ for flow visualization. The PIV flow field measurements were undertaken at two different locations: (i) upstream region, far from the dome location and (ii) around the dome region. An example of a PIV image is shown in figure 1b, which demonstrates the flow around the hemispheric obstacle. The flow was observed with a field of view of $90 \times 70 \mathrm{~mm}^{2}$. The tracers' density was 15-20 particles per interrogation area. A diode laser was used as the light source for PIV measurements having a maximum power output of $200 \mathrm{~W}$ at a wavelength of $532 \mathrm{~nm}$. A cylindrical lens $(f=28 \mathrm{~mm})$ was used to generate a light sheet of approximately $1 \mathrm{~mm}$ thickness.

The synchronization between laser light pulses and the camera was accomplished by transistor-transistor logic pulses from the synchronizer. The time interval between each pair of images was $200 \mathrm{~ms}$ with the pulse separation time adjusted to $10 \mathrm{~ms}$. Full-frame images of $1024 \times 1024$ pixels (figure $2 \mathrm{~b}$ ) were acquired and transferred to a computer via a frame grabber. Using the DAVIS FLOWMASTER software provided by the Lavision System (Germany), the 2D PIV image was divided into $32 \times 32$ pixels size sub-regions using a multi-grid correlation process with $50 \%$ overlap. The average particle velocities were calculated using 100 images coupled with the cross correlation method. The spurious vectors calculated based on the local median filtering were less than $3 \%$. No data smoothing was used for the calculation of the velocity measurements.

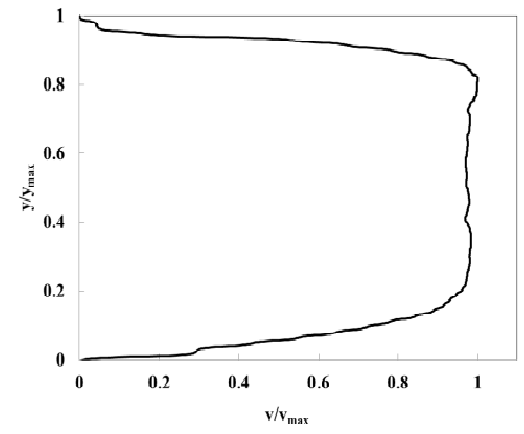

(a) Mean axial velocity at inlet.

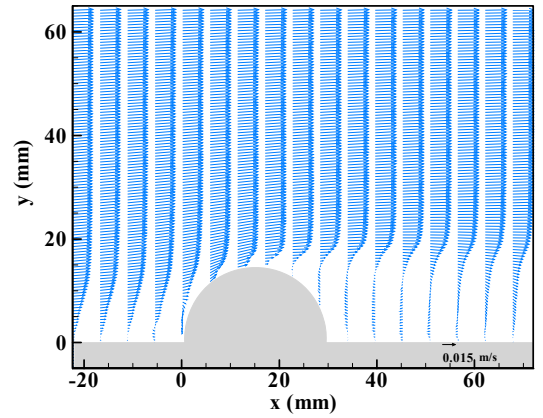

(b) Averaged axial velocity profiles around the dome.

Figure 2: $\quad$ Velocity measurements using PIV. 


\section{Results and discussions}

To verify the upstream flow conditions, a cross stream averaged velocity profile was obtained and is illustrated in figure $2 \mathrm{a}$. The velocity profile reached a maximum velocity between $0.2<y / y_{\max }<0.8$. The flow does represent a developing type. The boundary layers are visible at the top and bottom side of the channel.

In figure $2 b$, the large view of the averaged velocity profile around the dome is presented for the $\mathrm{Re}=800$. It is important to note that the flow is focused near the bottom wall of the channel and therefore the velocity does not reach its minimum value near top wall $(0<\mathrm{y}<60 \mathrm{~mm})$.

The magnitude of particle velocities is constant far from the hemisphere with an average velocity of $22 \mathrm{~mm} / \mathrm{s}$. Naturally and through the action of the pressure field as the fluid particles approach the hemisphere, their velocities decrease.

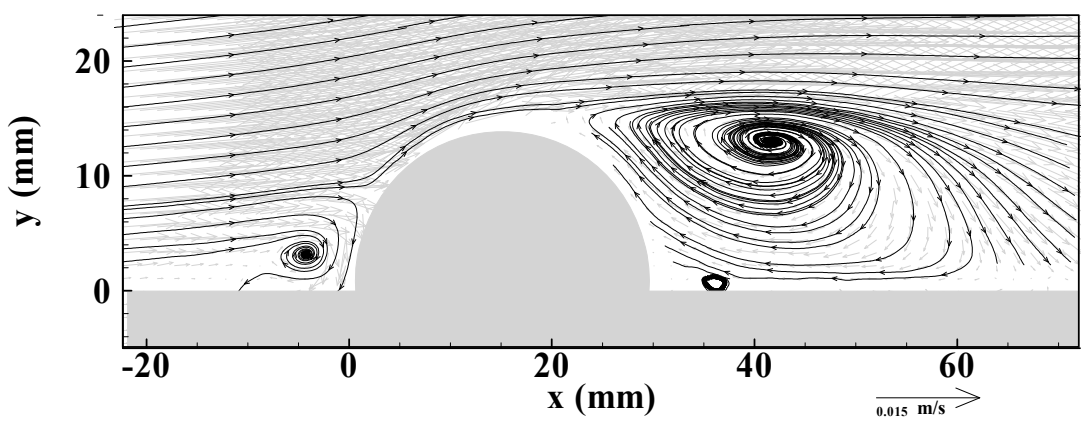

(a) Averaged axial velocity vector plot and its corresponding streamlines in a mid vertical plane for $\mathrm{Re}=800$.

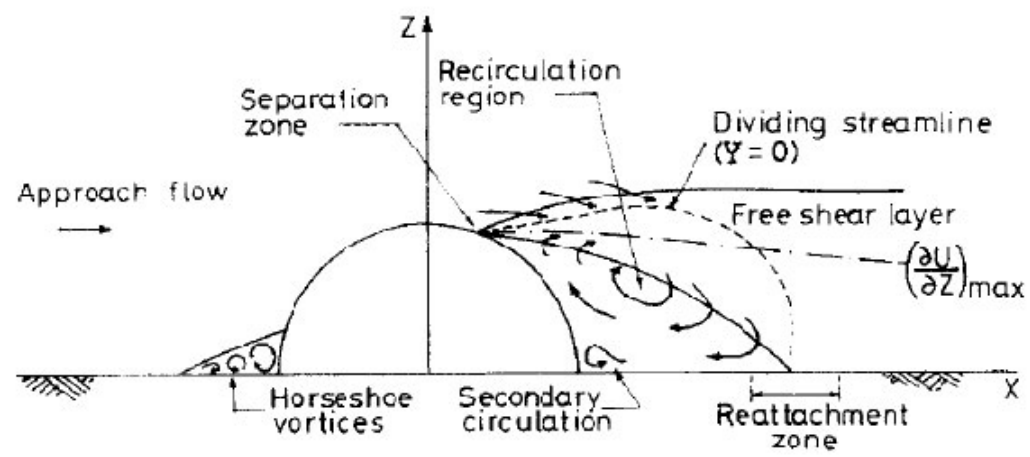

(b) Schematic flow characteristics at $\mathrm{Re}=1.4 \times 10^{5}$ [3].

Figure 3: Flow characteristics near the hemisphere, side view. 
Streamlines of the averaged velocity field, are presented in figure 3a, and compared with previous experimental observations reported by Savory and Toy [3], fig. 3b. In this paper the flow is laminar $(\operatorname{Re}=800)$. The averaged velocity profile shows parallel streamlines in the upstream region far from the dome obstacle (figure 3a). As the flow approaches the dome the streamlines are deviated in different directions. In lower layers behind the obstacle, the direction of the velocity changes. An upstream recirculating region similar to the previous observation [3] is denoted with a front stagnation streamline. Both the reattachment zone and the secondary circulation are clearly visible using the streamlines. The separation zone is located between ( $x=21 \mathrm{~mm}$ and $\mathrm{y}=12 \mathrm{~mm}$ ). Although similarities are observed between previous experimental results [3] and the present PIV measurements, fundamental differences exist such as the size and position of the recirculating zone. The PIV measurements indicate that the center of the recirculating zone is located both further downstream of the dome and at higher vertical position. This difference is due to the characteristics of the fluid flow (laminar and confined flow in this experiment versus turbulent and open flow in the previous study [3]). The other similarity which can be observed, is at the front side of the dome obstacle. Horseshoe vortices are shown in both cases, figs. 3a and b. However, in the present experiment the dimensions of the horseshoe vortices are larger than in the previous study of [3] and represent almost half of the dome diameter.

The magnitude of the vertical $\left(\mathrm{V}_{\mathrm{y}}\right)$ and horizontal $\left(\mathrm{V}_{\mathrm{x}}\right)$ velocities are illustrated in figures $4 \mathrm{a}$ and $4 \mathrm{~b}$ respectively. As shown in figure $4 \mathrm{a}$, the maximum axial velocity appears at $\mathrm{x}=10 \mathrm{~mm}$ and $\mathrm{y}=15 \mathrm{~mm}$, where the velocity is deviated from its original direction due to the presence of the dome as an obstacle. The negative velocities represented in dark blue color (or dark gray color in black and white print-B/W) correspond to the existence of horseshoe vortices. The negative and positive velocities are characterized by the dark blue (or dark gray in $\mathrm{B} / \mathrm{W}$ print) and red colors (or medium gray in $\mathrm{B} / \mathrm{W}$ print) respectively. The horizontal light blue region represents the thin boundary zone where the velocity is zero corresponding to the region where the main and recirculating flows are separated.

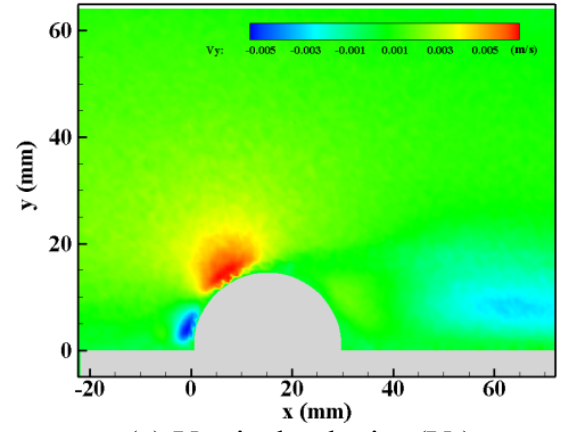

(a) Vertical velocity $\left(V_{y}\right)$.

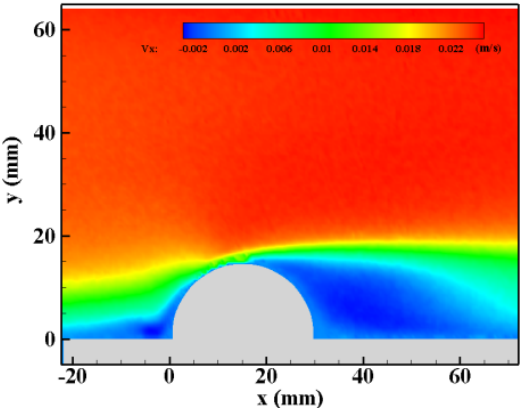

(b) Axial velocity $\left(\mathrm{V}_{\mathrm{x}}\right)$.

Figure 4: Velocity magnitude near the hemisphere, side view $(\operatorname{Re}=800)$. 
Figure 5a illustrates the streamlines around the dome in a horizontal plane near the bottom wall. In this case, since the laser is projected horizontally only part of the flow is optically accessible for measurements due to shadowing. The laser illuminates the horizontal flow field at $0.1 \mathrm{~mm}$ above the bottom wall as a camera records PIV pictures from the top view. Measurements are compared with the flow characteristics obtained by Savory and Toy [3] in which horseshoe vortices are observed around the dome. The reattachment is also clearly shown behind the dome. In particular, the bottom parts of the arch type downstream vortices mentioned above are captured by the PIV measurements. However, the flow field between two figures is slightly different. The horseshoe zone in front and around the dome is larger in the present experiment.

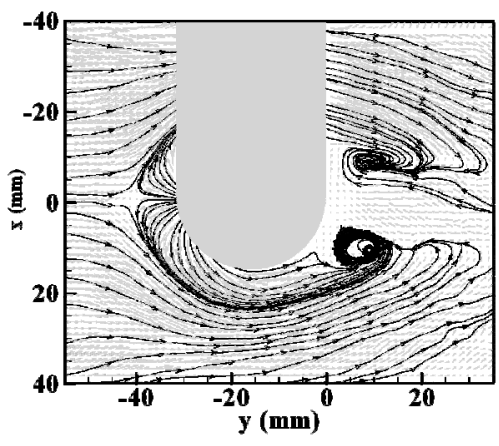

(a) Averaged velocity vector plot in a horizontal plane for $\mathrm{Re}=800$.

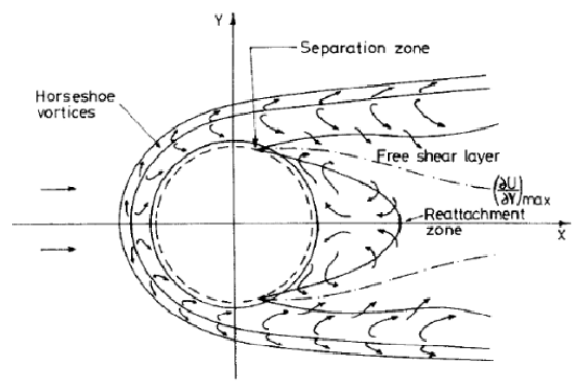

(b) Schematic flow characteristics at $\mathrm{Re}=1.4 \times 10^{5}[3]$.

Figure 5: Flow characteristics in a horizontal plane near the hemisphere bottom wall.

The PIV measurements show that the flow is highly three dimensional around the dome. It was also observed that flow inside the horseshoe vortices and reattachment zone is not stable. The location of the separation zones fluctuate with time between two instantaneous images. However, the averaged velocity shows a structure and a quasi-symmetric flow in the horizontal plane.

\section{Conclusions}

Laminar flow past a smooth hemisphere was investigated using the Particle Image Velocimetry technique. Vertical and horizontal 2D velocity distributions at $\mathrm{Re}=800$, showed a laminar flow. The averaged velocity distributions around the hemispherical obstacle were obtained and velocity field illustrated the presence of a complex three dimensional flow containing intriguing structures such as a horseshoe vortex enveloping the dome and arch-shaped vortices in the 
downstream region. The location of the reattachment point, the separation line, and the reverse flow are also identified and discussed. The characteristics of laminar flow around the dome share several similar features with the case of turbulent flow [3] with significant difference in the size of vortices and their location. The results of this research can be employed to validate further numerical predictions of this complex flow field. Future experiments will focus on the influence of the dome surface roughness and velocity distribution of turbulent flows around the hemispherical obstacle.

\section{References}

[1] Acarlar, M.S. and Smith C.R., A study of hairpin vortices in a laminar boundary layer, Part1. Hairpin vortices generated by a hemisphere protuberance. J. Fluid Mech., 175, pp. 1-41, 1987.

[2] Taniguchi, S., Sakamoto, H., Kiya, M. and Arie, M., Time-averaged aerodynamic forces acting on a hemisphere immersed in a turbulent boundary. J. Wind Eng. Ind. Aerod., 9, pp. 257-273, 1982.

[3] Savory, E. and Toy, N., Hemispheres and hemisphere-cylinders in turbulent boundary layers. J. Wind Eng. Ind. Aerod., 23, pp. 345-364, 1986.

[4] Cheng, C.M. and Fu, C.L., Characteristic of wind loads on a hemispherical dome in smooth flow and turbulent boundary layer flow. J. Wind Eng. Ind. Aerod., 98, pp. 328-344, 2010.

[5] Tamai, N., Asaeda, T. and Tanaka, N., Vortex structures around a hemispheric hump. Bound-Lay. Meteorol., 39, pp. 301-314, 1987.

[6] Tavakol, M.M., Yaghoubi, M. and Masoudi Motlagh, M.M., Air flow aerodynamic on a wall mounted hemisphere for various turbulent boundary layers. Exp. Thermal and Fluid Sci., 34, pp. 538-553, 2010.

[7] Kim, D. and Choi, H., Laminar flow past a hemisphere. Phy. of Fluids, 15(8), pp. 2457-2460, 2003. 